\title{
UAV autonomous path optimization simulation based on radar tracking prediction
}

\author{
Bo Wang ${ }^{*}$, Jianwei Bao, Li Zhang and Qinghong Sheng
}

\begin{abstract}
In the UAV path planning study, due to the relative movement of multiple targets and the UAV, long-term and large-scale UAV, autonomous tracking has not been achieved. Therefore, aiming at this problem, this paper uses radar to provide a real-time feedback on target position, estimates the later motion state of the target according to its position, and then perform dynamic path planning by combining the feedback data and the state estimation result. Finally, The UAV path is optimized in real time. Experiments show that the proposed scheme can better plan the UAV path when multiple targets are in motion, thus improving the intelligence of the UAV and the capability of long time tracking.
\end{abstract}

Keywords: UAV, Radar target tracking, Motion estimation, Path planning

\section{Introduction}

With the development of UAV technology, UAVs have achieved a wide application in the field of military and civil aviation remote sensing, communications relay, and so on [1]. At the same time, with the increase of the UAV payload and the improvement of the radar miniaturization technology, the UAV radar has gradually become an important part of the UAV load.

At present, the UAV radar is mainly composed of synthetic aperture radar, and the working frequency bands are concentrated in the millimeter wave band (30 300 GHz), the Ku band (12 18 GHz), and the $\mathrm{X}$ band $(8 \sim 12 \mathrm{GHz})$ [2]. UAV SAR has high resolution and long detection range. It can work all day and around the clock, penetrate clouds and obstacles, and track moving targets and sudden rapid maneuvering targets. Because of the strong anti-interference ability and around-the-clock application of radar, the UAV can use radar sensors to obtain environmental information during flight and process it through signal processing algorithms and target tracking technology, which provides reliable environmental information for UAVs.

Radar is an important sensor in the field of target tracking and recognition. The electromagnetic radiation has strong reliability, but it also has some shortcomings. Due

\footnotetext{
* Correspondence: wangbo_nuaa@nuaa.edu.cn

College of Astronautics, Nanjing University of Aeronautics and Astronautics, NO. 29 Yudao Street, Nanjing 210016, Jiangsu province, China
}

to the complex electromagnetic environment in the low-altitude airspace, the clutter signal noise is relatively strong, which will seriously affect the radar's ability to detect moving targets. Compared with the infrared imaging system, the visible light sensor has strong denoising ability and imaging capability. It can provide clearer image information and is less susceptible to electromagnetic interference. The radar target tracking is based on the measurement information of the point target for filtering and tracking, and the visible light sensor tracking is based on the image matching degree $[3,4]$. In the field of UAV target tracking, a hot research trend is how to combine the advantages of point target tracking and image tracking together to solve the problem of imaging blur of simple image targets at long distances and the interference of close-range targets by low-altitude airspace.

At present, in the aspect of moving target tracking by UAV, the existing autonomous tracking of the single moving target has been extensively studied and applied. However, as to the problem of multiple moving target tracking, the UAV operation still relies on operator's observing in order to control the UAV flight path, and the autonomous flight path design has not been realized. Therefore, this paper combines the tracking, prediction, and path planning of single moving target by UAV, so as to realize UAV autonomous path planning for multiple targets.

In order to achieve long-term and large-scale UAV autonomous tracking of multiple moving targets, this 
paper studies the UAV autonomous optimization based on multiple moving target tracking. There are $\mathrm{n}$ moving targets to be tracked, and a UAV starts from a point to reach the positions of these targets. After each target is visited once, the UAV will return to the starting point. The UAV path problem means how to find the shortest path of the process above. In order to realize the UAV autonomous path planning [5], this paper divides the scheme into three steps.

The first step is to obtain the specific motion equation of the moving targets after selected. Because of the continuous relative movement of the UAV and the targets, coordinates of the targets will constantly change and need to be updated in real time. In order to obtain the motion equation of the moving targets, the positions of the targets are obtained by radar in this paper, and the interception, tracking, track calculation, and tracking state determination of the targets are completed by radar data processing. In radar data processing, the motion trajectory of the target is established through correlation and filtering processing, which effectively eliminates false detection caused by residual clutter and updates the target position.

The second step is to predict the positions of moving targets. Because the position of a target is constantly changing, tracking its present position will lead to longer tracking time. Therefore, this paper uses the method of estimating the target position to optimize the UAV path. Due to the state change and uncertainty of moving targets, Kalman filtering method [6, 7] is used in this paper. In the radar target tracking filtering algorithm, Kalman filtering is the most representative, which is both based on the measurement information of the point target and predicting the position of the target at the next moment to achieve tracking $[8,9]$. Kalman filtering is a recursive algorithm for solving the state optimal estimation. It can update the current state and predict the state of the next moment by filtering only the state information of the previous moment. This tracking algorithm is characterized by small calculation and nice real-time ability $[10,11]$. And the Kalman filtering method [12] is a time domain method which solves the problem of optimal filtering based on state space. It has high applicability to the estimation of moving targets that frequently change motion states.

The last step is to use predicted positions to dynamically plan the UAV path, which means to quickly and accurately design a non-repeating shortest path after obtaining the specific positions of the targets. This problem is called the traveling salesman problem (TSP) in the unified research $[13,14]$. Due to the complexity of TSP, when the scale of targets is large, the time complexity of the exact solution method will increase exponentially, and it will lead to high the real-time requirements during the operation of the UAV. The number of targets is considered to be about 10 , and the universality of the algorithm should be considered. Therefore, ant colony algorithm is adopted in this paper instead of the exact algorithm to obtain the approximate shortest path optimal solution. The basic principle of the ant colony algorithm is to imitate the ant colony foraging behavior in the natural world, use a pheromone to control the direction of ant's movement, and autonomously and effectively approach the optimal path ultimately [15]. However, although the basic ant colony algorithm has a strong global optimization ability, it takes a long time to solve the problem, and it is easy to fall into a local optimum and cause the algorithm to stall. To solve this, Stutzle and Hoos [16] proposed the maximum and minimum ant colony algorithm. Only the pheromone of the ant with the optimal algorithm is updated; thus, the convergence speed of the algorithm is improved. Li et al. [17] use prior knowledge to limit the increment of pheromone in the ant colony algorithm and increase the road weight factor, so as to effectively avoid the stagnation of the algorithm. Therefore, this paper combines the improved methods of scholars to optimize the ant colony algorithm, thus solves the problem of long solution time and falling into the local optimum in the route plan using ant colony algorithm.

In general, in order to solve the problem of UAV autonomous flight path planning, this paper improves the method of UAV single moving target. Firstly, radar is used to track multiple moving targets to obtain the real-time position and motion information of multiple moving targets. Then, the Kalman filter method is used to predict and estimate the positions of the moving targets. Finally, the improved ant colony algorithm is used to process the acquired information to design a shortest UAV flight path that can track multiple moving targets. The scheme can realize the near-optimal UAV flight path by means of computer vision under the scene of UAV multiple moving targets tracking, thereby reducing manual operations and improving the UAV autonomous flying capability.

\section{Algorithm implementation \\ 2.1 Overall algorithm flow}

This paper presents an algorithmic process for UAV autonomous planning. Firstly, the radar sensor mounted on the UAV is used to obtain the positions of the moving targets and their changes and then estimates the positions of the targets after a certain period of time according to the changes of their positions. After obtaining the estimated motion states, the shortest path plan under the estimated target position obtained by using the improved ant colony algorithm. Finally, when the UAV performs the calculated path flight, it updates the positions of the targets and the path in real time to achieve the UAV path planning requirement for multiple moving targets. The specific flow chart is shown in Fig. 1. 


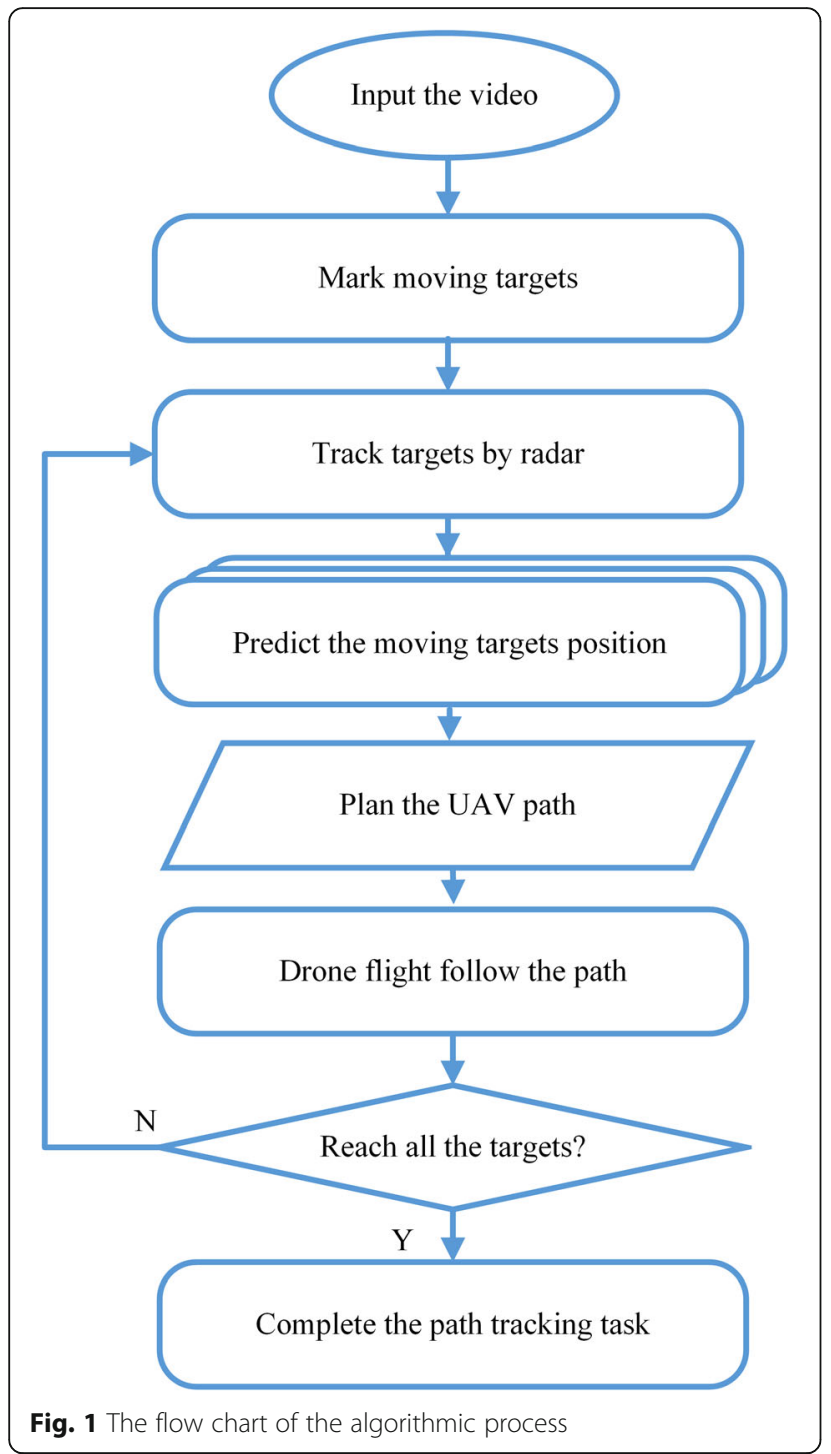

\subsection{Radar target location and prediction}

Radar target location plays a crucial role in radar target tracking. However, there is a state noise in the target environment and system noise in the radar system. These two kinds of noise can cause an inevitable error between the true state and the measured state of the cloud target. In order to eliminate this error, a lot of research has been carried out at home and abroad, mainly to develop filtering methods [18], such as Kalman filtering.

Kalman filter is a recursive estimator that implements the estimation of motion states through the principle of feedback control [19]. After observing the positions of the targets by radar, the current states of the targets can be predicted according to the historical motion states of the targets. The basic state model of the targets includes the observation equation and the state equation as follows:

$$
\left\{\begin{array}{l}
Z_{k}=H_{k} X_{k}+V_{k} \\
X_{k, k-1}=A_{k, k-1} X_{k-1}+B_{k-1} U_{k-1}
\end{array}\right.
$$

Where $Z_{k}$ represents the observed value of the target state, $H_{k}$ represents the observation matrix, $X_{k}$ represents the real state at time $k, X_{k, k-1}$ represents the target state estimated from the previous state, $A_{k, k-1}$ represents the state transition matrix from $k-1$ to $k, B_{k}$ ${ }_{-1}$ represents the system control matrix at time $k-1, U_{k}$ ${ }_{-1}$ represents the noise that influences the current motion law of the targets such as acceleration and deceleration of the moving targets, $Q$ is the self-covariance of $U_{k-1}$, and $V_{k}$ represents the observation noise, which in this experiment represents the deviation during the tracking process, and $R$ is the covariance of $V_{k}$.

The flow of the Kalman filter in each frame is shown in Fig. 2.

Therefore, the observation states of the radar can be filtered by the above formula to obtain the state equation of the targets, because the UAV takes a long time in the process of approaching the target. If only relying on the existing states of the targets, the direct interval of the control signal and the delay in the signal transmission may cause the flight direction to be updated backward, so that the shorter tracking path cannot be obtained. Thus, in this paper, the predicted positions in the Kalman filter algorithm are extracted in order to take control of the UAV.

From the Kalman filter formula, the prediction equation of the next coordinate $X_{k+1, k}$ and its covariance matrix $P_{k+1, k}$ can be obtained as:

$$
\left\{\begin{array}{l}
X_{k+1, k}=A_{k+1, k} X_{k}+B_{k} U_{k} \\
P_{k+1, k}=A_{k+1, k} P_{k} A_{k+1, k}^{T}+B_{k} Q_{k} B_{k}^{T}
\end{array}\right.
$$

In this paper, the principle of optimizing the algorithm path through the Kalman filter is shown in Fig. 3. If the prediction algorithm is not added, due to the delay of the UAV flight control signal, the UAV will move towards the direction of the obtained target position and reach position 3 . At the same time, the target will move during the flight of the UAV and reach position 2 . Therefore, the prediction algorithm added in this paper corrects the flight direction of the UAV and makes the UAV reach position 4 where it is close to the target position, when it gets the target position next time.

\subsection{UAV path planning}

Taking into account the real-time requirement of UAV path planning, the ant colony algorithm is used. But the ant colony algorithm is slow in convergence and easy to fall into the local optimal solution [20]. So, this paper focuses on the optimization of ant colony algorithm. The probability of stagnation of the algorithm is reduced by 

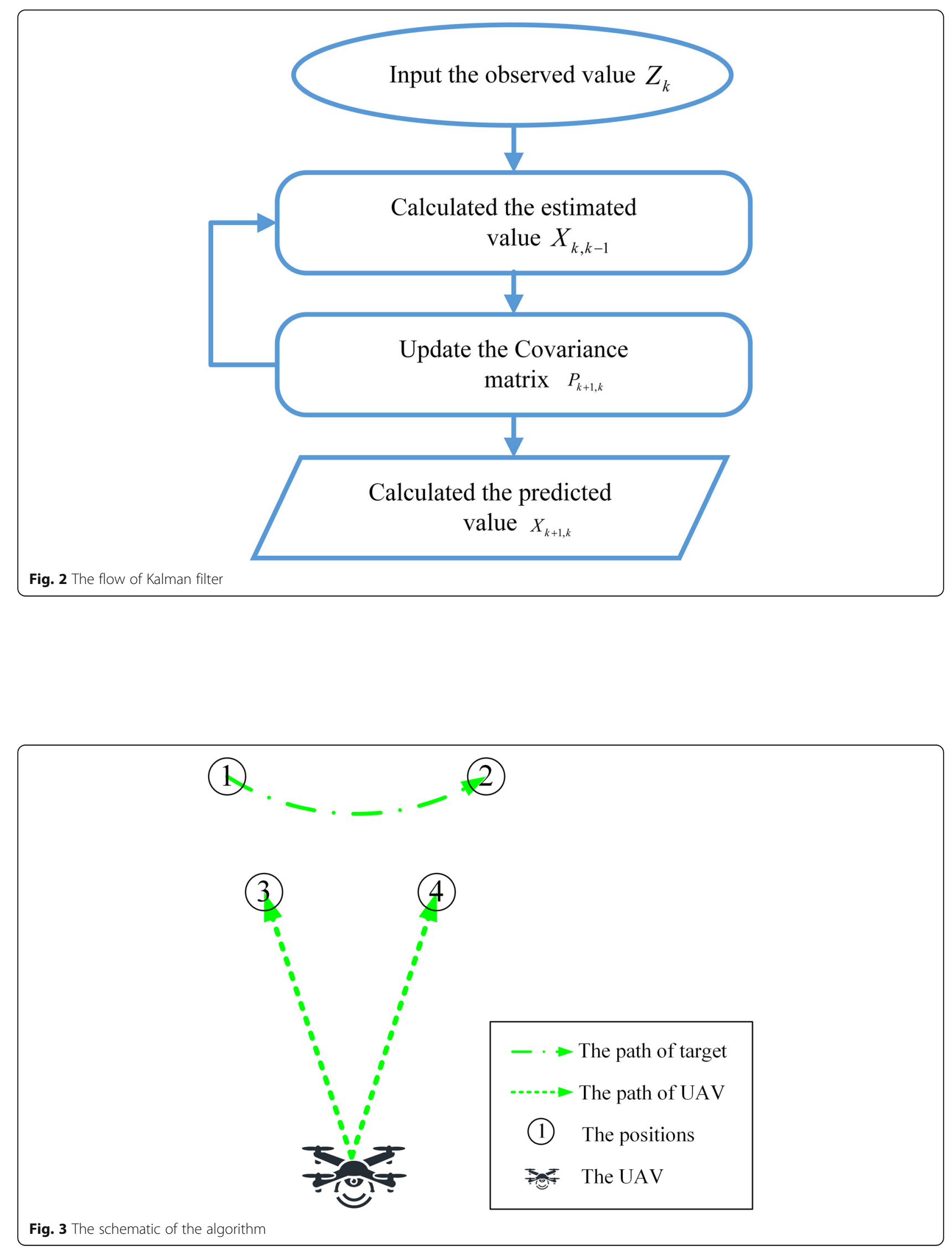
limiting the increment of pheromone update and the dynamic change of volatile factors; thus, the UAV dynamic path planning can be realized.

Firstly, information is initialized for each target:

$$
\tau_{i j}(0)=m / d_{\min }
$$

where $\tau_{i j}(0)$ represents the pheromone from target $i$ to target $j, m$ represents the number of ants, and $d_{\min }$ represents the distance between the closest two targets.

Then, the ants transfer on the basis of the pheromone:

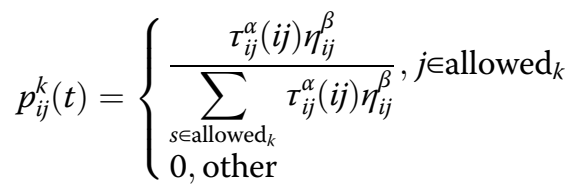

Where $p_{i j}^{k}(t)$ represents the probability that ant $k$ transfers from city $i$ to city $j ; \eta_{i j}(t)$ represents the concentration of pheromone on the path at time $t ; \eta_{i j}(t)=1 / d_{i j}$ represents the degree of expectation of ants from target $i$ to target $j ; \alpha$ represents the information heuristic factor, which indicates the influence of pheromone on the transition probability; $\beta$ represents the expected heuristic factor, which indicates the influence of visibility on the transition probability of ants; allowed $=\{1,2, \ldots, n\}-$ tabu $_{k}$ represents the targets which ant $k$ has not visited; and tabu $k_{k}$ represents the targets that the ant $k$ has passed.

Then update of pheromone partially:

$$
\tau_{i j}(t+1)=\left\{\begin{array}{l}
(1-\rho) \tau_{i j}(t)+\varepsilon\left(L_{\text {worst }}-L_{\text {best }}\right), \text { best path. } \\
(1-\rho) \tau_{i j}(t)-\varepsilon\left(L_{\text {worst }}-L_{\text {best }}\right), \text { worst path } \\
(1-\rho) \tau_{i j}(t), \text { other }
\end{array}\right.
$$

where $\rho$ represents the pheromone volatility coefficient, $\varepsilon$ represents the pheromone enhancement coefficient, and $L_{\text {best }}, L_{\text {worst }}$ represent the length of the optimal path and the worst path, respectively, for all ants in this cycle. In order to increase the size difference of the pheromone between the optimal path and the worst path, this paper adopts the maximum and minimum ant system to strengthen the optimal solution and weaken the worst solution.

To avoid too much pheromone difference caused by the maximum and minimum ant system, which will lead to the stagnation of the algorithm, the amount of all pheromone should be limited as:

$$
\tau_{i j}(t)=\left\{\begin{array}{l}
\tau_{\max }, \text { if } \tau_{i j}(t)>\tau_{\max } \\
\tau_{i j}(t), \text { if } \tau_{\min }<\tau_{i j}(t)<\tau_{\max } \\
\tau_{\min }, \text { if } \tau_{i j}(t)<\tau_{\min }
\end{array}\right.
$$

where $\tau_{\max }$ represents the maximum pheromone value; $\tau_{\min }$ represents the maximum pheromone value.

Finally, the ant colony will gradually converge on the best path through the constant updating of pheromone.
If the number of cycles is small at this time, the ant colony path will be modified by the crossover and mutation of the genetic algorithm, so the pheromone can be updated again. Thereby, it can reduce the occurrence of the local optimal solution.

The specific process is shown in Fig. 4.

The experimental principle of this paper is shown in Fig. 5. By predicting the position of each target, the UAV re-adjusted the tracking sequence to the targets and adjusted the first tracking target from the no. 1 target far away from the UAV to the no. 2 target approaching the UAV, thus reducing the total path length of UAV. So, the planned path is more adaptive to the targets' movement.

\section{Simulation experiment and results}

\subsection{The experiment of Kalman prediction algorithm}

At first, there is the simulation experiment of Kalman prediction in the paper to verify the accuracy of the prediction algorithm. There is a target which moving along a sinusoidal trajectory. And the target will give a feedback position for every 10 frames to simulate the radar. And the Kalman prediction algorithm will predict the position of the target 10 frames later. And the current position of the target will be compared with the predicted position before 10 frames. The experimental results are shown in Fig. 6.

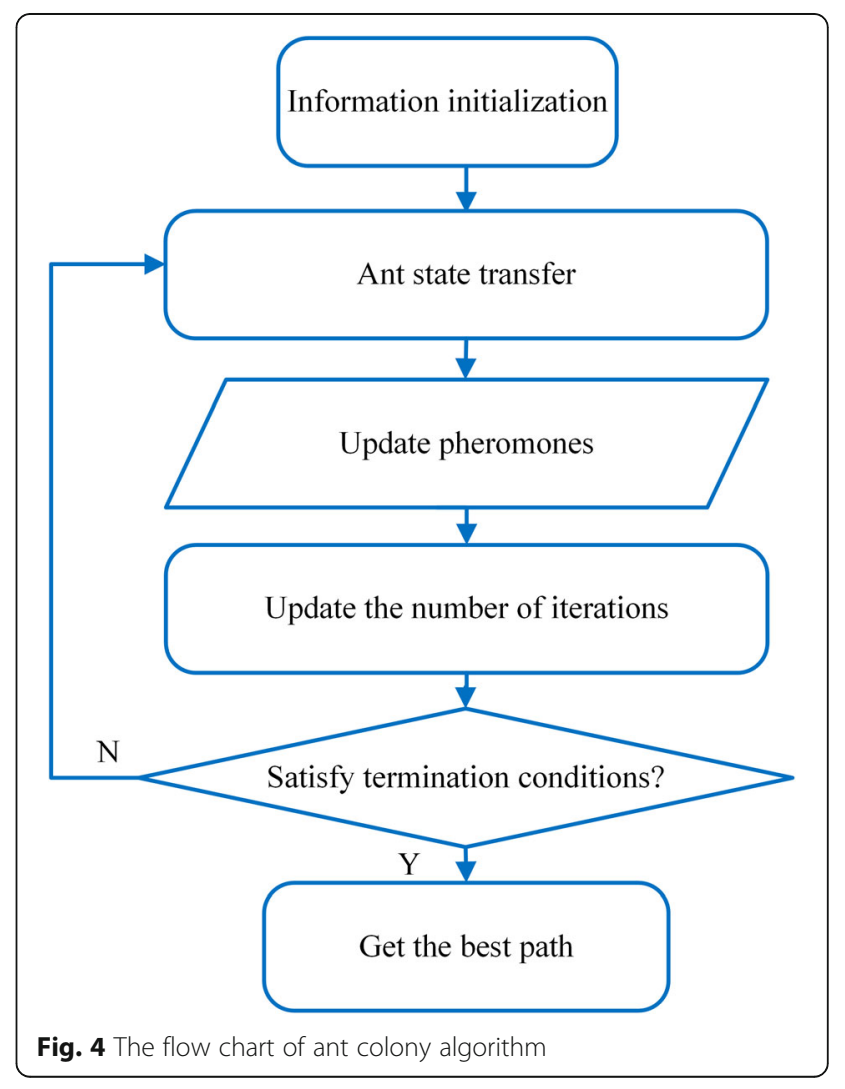




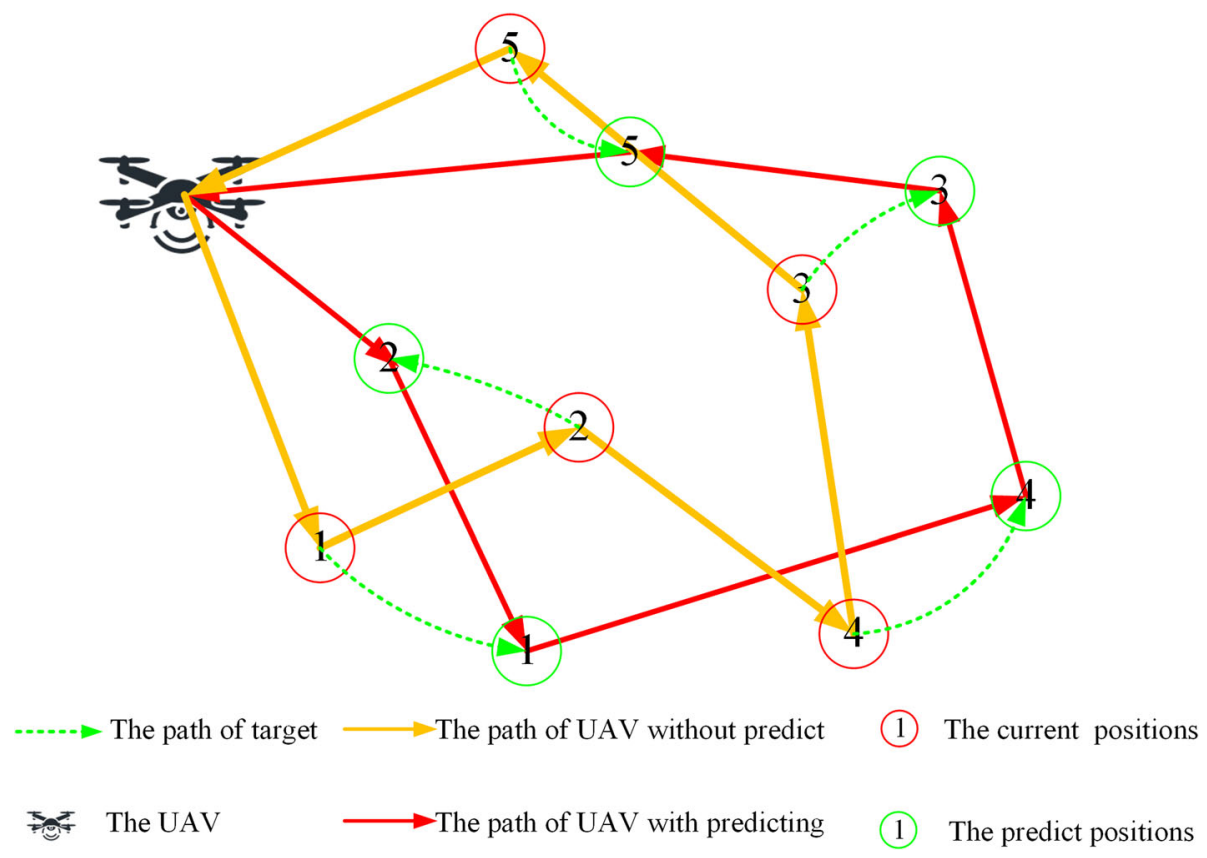

Fig. 5 The schematic diagram of the path planning method

\subsection{Single moving target tracking experiment}

In order to prove the validity and feasibility of the algorithm, simulation experiments on single target tracking and multi-target tracking of the UAV are conducted.

First of all, a simulation experiment of radar single target tracking is conducted. The UAV radar sensor is used to obtain the observation position of the target, and the Kalman filter method is used to predict the position of the moving target. The experiment adopts two kinds of UAV tracking methods, target state position tracking and target predicted position tracking. In this paper, the UAV of target state position tracking is called the comparison UAV, and the UAV of target predicted position tracking is called the experimental UAV. In the experiment, the speed of the UAV is set to two pixels per frame, and the target moving speed is about one pixel per frame. The experimental results are shown in Fig. 7.

\subsection{Multiple moving targets experimental verification}

Then, a simulation experiment for the UAV path planning of multi-moving target tracking is conducted. Based on the radar obtaining the position of a single moving target, multiple moving targets are observed. After that, according to the states of the targets, the ant colony
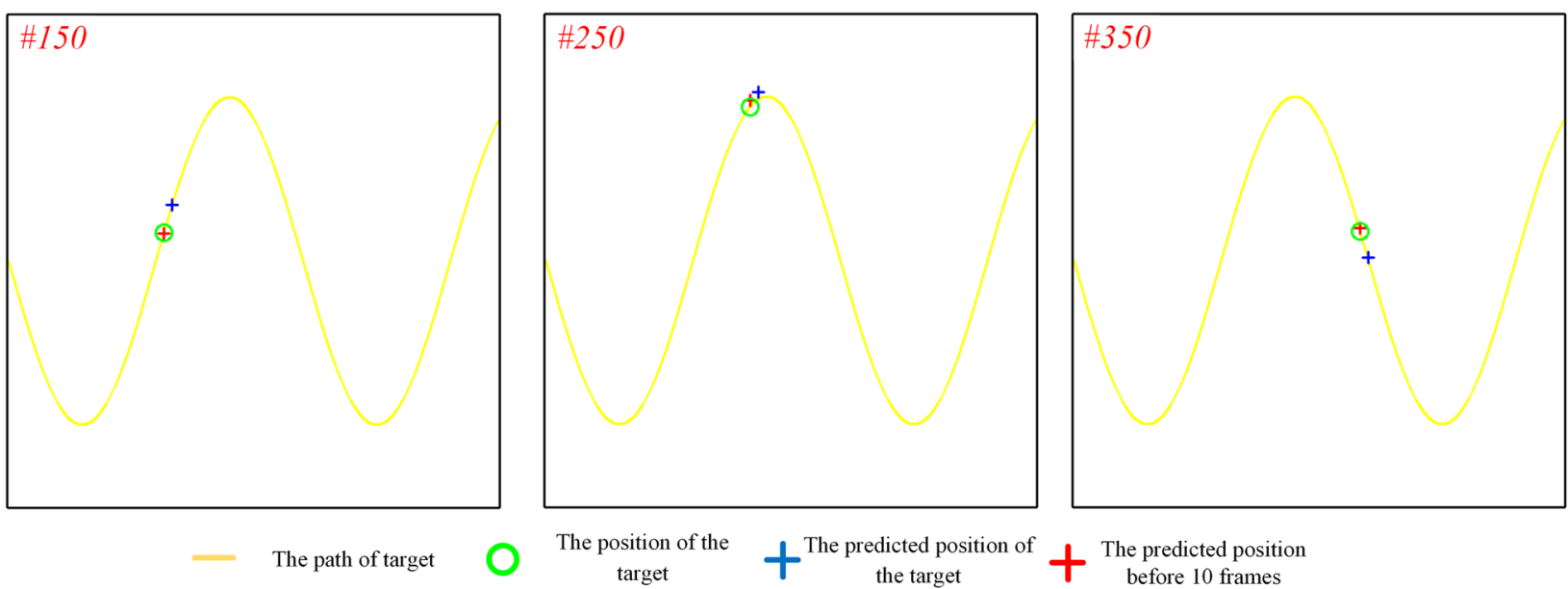

Fig. 6 The experimental results of the Kalman prediction algorithm 


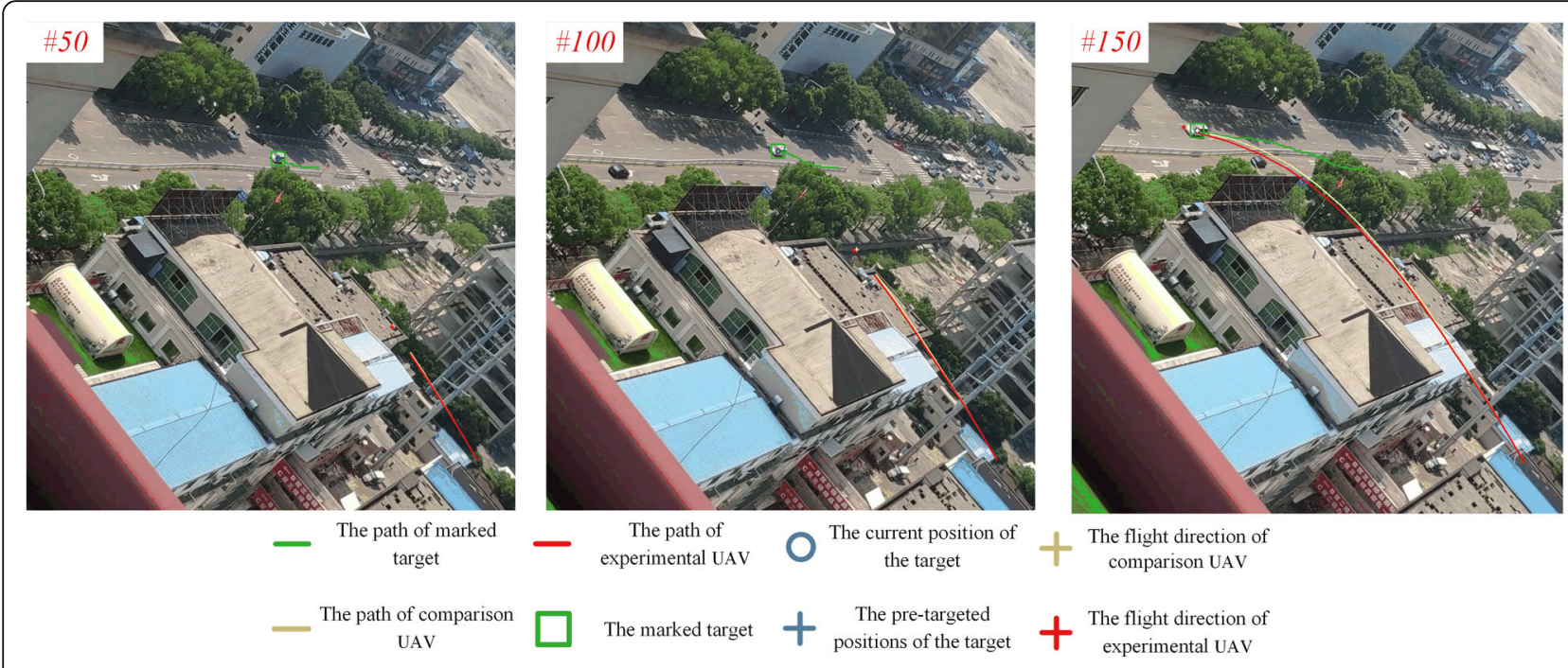

Fig. 7 The experimental results of the simulation of a single moving target

algorithm is used to determine the target tracking sequence and path. The simulation results are shown in Fig. 8.

\section{Discussion of the results}

4.1 Discussion of the Kalman prediction algorithm experiment As shown in Fig. 6, in the 150th and 350th frames, when the target motion state changes a little, the predicted position before 10 frames can coincide with the current position of the target, and it can still be above the trajectory of the target's motion. And in the 250th frame, when the target is in the case of a large movement direction change, the Kalman prediction algorithm can also be adjusted quickly with only minor deviations. It means that the Kalman prediction algorithm can effectively predict the target position.

\subsection{Discussion of single moving target tracking experiment} Figure 7 shows that by predicting the target position according to the state of the observed target by radar, the experimental UAV makes a judgment on the target motion direction and corrects the direction in advance, thereby reducing the path length required to reach the target and shortening the time required to get close to the target. The tracking efficiency is improved and the path planning is optimized.

\subsection{Discussion of multiple moving targets experimental verification}

As is shown in the 60th frame of Fig. 8, the experimental UAV modifies the flight path and the tracking sequence, and in the 70th frame, after 10 frames, the comparison UAV is modified for the moving target tracking sequence to the same as the experimental UAV. It shows that the proposed algorithm can better adapt to the motion changes of multiple moving targets, track the moving targets, and update and correct the UAV path in advance, thus improving the tracking efficiency of the UAV.
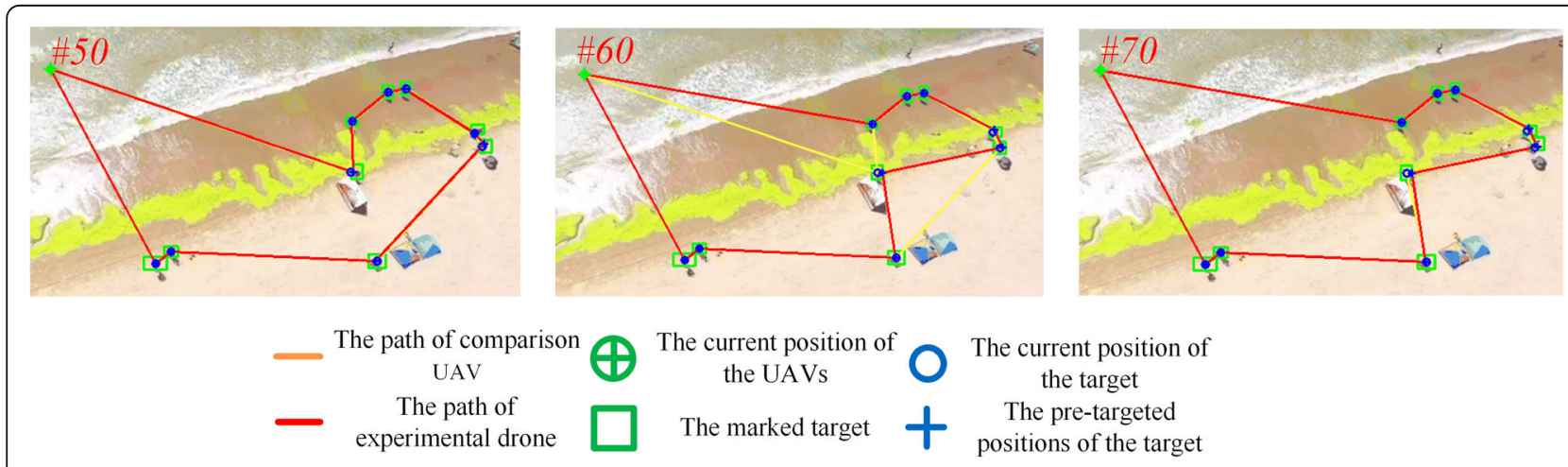

Fig. 8 The experimental results of the simulation of the path planning of multiple targets 


\section{Conclusions}

In this paper, aiming at the problem of UAV path planning for multiple moving targets, after the targets are determined, radar is used to observe the targets and obtain their observation positions. According to the observation positions of the targets, the Kalman filtering is used to obtain the state positions and the predicted positions of the targets, and the ant colony algorithm is used to perform path planning for multiple moving targets. The experimental results show that by predicting the position of the moving targets for path planning, the UAV path can be corrected and updated in advance, which improves the tracking efficiency of the UAV, thus enhancing the intelligence of the UAV multiple moving targets tracking.

\section{Abbreviations}

TSP: Traveling salesman problem; UAV: Unmanned aerial vehicle

\section{Funding}

This work was supported by the "Fundamental Research Funds for the Central Universities," no. NS2017062.

\section{Availability of data and materials}

The datasets used and/or analyzed during the current study are available from the corresponding author on reasonable request.

\section{Authors' contributions}

BW, JB, LZ, and QS conceived the idea of the paper. BW and JB designed and performed the experiments. BW, JB, and BW analyzed the data. LZ and QS wrote the paper. BW, JB, LZ, and QS revised the paper. All authors read and approved the final manuscript.

\section{Competing interests}

The authors declare that they have no competing interests.

\section{Publisher's Note}

Springer Nature remains neutral with regard to jurisdictional claims in published maps and institutional affiliations.

Received: 8 August 2018 Accepted: 28 September 2018

Published online: 11 October 2018

\section{References}

1. X. Wang, Vision-based detection and tracking of a mobile ground target using a fixed-wing UAV. Int. J. Adv. Robotic Syst. 11(156), 1-11 (2014). https://doi.org/10.5772/58989

2. L. Liu, J.I. Bo, Status and development of radar on UAV. Mod. Nav. 3, 227-230 (2014)

3. Y. Zhang, S. Hu, Research on radar/visible light surveillance and tracking methods of target in low altitude airspace. Comput. En. Appl. 54(6), 234-240 (2018). https://doi.org/10.3778/j.issn.1002-8331.1610-0149

4. X. Liu, F. Li, Z. Na, Optimal resource allocation in simultaneous cooperative spectrum sensing and energy harvesting for multichannel cognitive radio. IEEE Access. 5, 3801-3812 (2017). https://doi.org/10.1109/ACCESS.2017.2677976

5. P. Han, M. Chen, S.D. Chen, et al., Path planning for UAVs based on improved ant colony algorithm. J. Jilin Univ. 31(1), 66-72 (2013). https://doi. org/10.3969/j.issn.1671-5896.2013.01.011

6. R.E. Kalman, A new approach to linear filtering and prediction problems. Trans. ASME J. Basic Eng. 82(1), 35-45 (1960). https://doi.org/10.1115/1.3662552

7. G.Y. Kulikov, M.V. Kulikova, Accurate cubature and extended Kalman filtering methods for estimating continuous-time nonlinear stochastic systems with discrete measurements. Appl. Numer. Math. 111, 260-275 (2017). https:// doi.org/10.1016/j.apnum.2016.09.015

8. S. Chen, H. Liu, J. Chen, L. Shen, Penetration trajectory planning based on radar tracking features for UAV. Aircr. Eng. Aerosp. Tec. 85(1), 62-71 (2013). https://doi.org/10.1108/00022661311294067
9. X. Liu, M. Jia, X. Zhang, W. Lu, A novel multi-channel Internet of Things based on dynamic spectrum sharing in $5 \mathrm{G}$ communication. IEEE Internet Things. 99, 1 (2018). https://doi.org/10.1109/JIOT.2018.2847731

10. X. Wang, R. Wang, N. Li, H. Fan, et al., A method of estimating the velocity of moving targets for use in high-resolution wide-swath SAR imaging. Remote Sens. Lett. 9(4), 305-313 (2018). https://doi.org/10.1080/2150704X. 2017.1420263

11. X. Liu, M. Jia, Z. Na, W. Lu, et al., Multi-modal cooperative spectrum sensing based on Dempster-Shafer fusion in 5G-based cognitive radio. IEEE Access. 6, 199-208 (2018). https://doi.org/10.1109/ACCESS.2017.2761910

12. A. Ahmadreza, K. Ali, M. Ali, An IMM algorithm based on augmented kalman filter for maneuvering target tracking. Sci. Res. Essays. 6(34), 6787-6797 (2011). https://doi.org/10.5897/SRE10.980

13. G. Dantzig, S. Johnson, Solution of a large-scale traveling-salesman problem. Oper. Res. 2(4), 393-410 (2010). https://doi.org/10.2307/166695

14. M. Manfrin, M. Birattari, T. Stützle, et al., Parallel ant colony optimization for the traveling salesman problem. Lect. Notes Comput. Sci. 4150(2), 224-234 (2016). https://doi.org/10.1002/j.1538-7305.1965.tb04146.x

15. M. Dorigo, V. Maniezzo, A. Colorni, Ant system: optimization by a colony of cooperating agents. IEEE T. Syst. Man. Cy. B. 26(1), 29 (1996). https://doi.org/ $10.1109 / 3477.484436$

16. T. Stutzle, H. Hoos, MAX-MIN Ant System and local search for the traveling salesman problem. IEEE Int. Conf. Evol. Comput., 309-314 (2002). https://doi. org/10.1109/ICEC.1997.592327

17. S. Li, Y. Zhang, Y. Gong, The research on the optimal path of intelligent transportation based on ant colony algorithm. J. Changchun Univ. Sci. Tech. 4, 122-126 (2015). https://doi.org/10.3969/j.issn.1672-9870.2015.04.027

18. S.L. Sun, Z.L. Deng, Multi-sensor optimal information fusion Kalman filter. Aerosp. Sci. Technol. 40(6), 1017-1023 (2004). https://doi.org/10.1016/j. automatica.2004.01.014

19. B. Chen, S. Zhao, P. Zhu, J.C. Principe, Quantized kernel least mean square algorithm. IEEE Trans. Neural Netw. Learn. Syst 23, 22-32 (2012). https://doi. org/10.1109/TNNLS.2011.2178446

20. H.M. Botee, E. Bonabeau, Evolving ant colony optimization. Adv. Complex Syst. 1(2), 149-159 (1998). https://doi.org/10.1142/S0219525998000119

\section{Submit your manuscript to a SpringerOpen ${ }^{\circ}$ journal and benefit from:}

- Convenient online submission

- Rigorous peer review

- Open access: articles freely available online

High visibility within the field

Retaining the copyright to your article

Submit your next manuscript at $>$ springeropen.com 